\title{
On the Leitmann Equivalent Problem Approach
}

\author{
F.O.O. Wagener
}

Published online: 5 March 2009

(C) The Author(s) 2009. This article is published with open access at Springerlink.com

\begin{abstract}
The purpose of this paper is to show how Leitmann's equivalent problem approach ties in with the classical notions of the calculus of variations and how it can be exploited to give a rapid and elegant approach to the Weierstrass theory of sufficient conditions. Fixed, free and constrained endpoint conditions are considered.
\end{abstract}

Keywords Calculus of variations · Equivalent problems · Rectifying coordinates · Sufficient conditions

\section{Introduction}

George Leitmann has introduced a method of transforming a calculus of variations problem into an equivalent, and possibly simpler, form $[1,2]$. This method consists of performing a coordinate transformation of the state space, followed by a transformation to an equivalent variational problem, in the sense of Carathéodory, by subtracting a null Lagrangian, that is the total derivative of a function that depends on time and space. Carlson and Leitmann have recently pointed out that the equivalent problem takes a particularly simple form if the coordinate transformation is furnished by a field of extremals $[3,4]$; in fact, they obtain the so-called Weierstrass representation formula (see [5], Chap. 6, p. 333) with a particularly simple form of the Weierstrass excess function.

Communicated by D.A. Carlson.

The author thanks Dean Carlson, George Leitmann and Saeed Mohammadian Moghayer for helpful remarks and discussions. This work has been supported by NWO under a MaGW-VIDI grant.

F.O.O. Wagener $(\bowtie)$

CeNDEF, Department of Quantitative Economics, University of Amsterdam, Roetersstraat 11, 1018 WB, Amsterdam, Netherlands

e-mail: F.O.O.Wagener@uva.nl 
The elements of this approach are well known. Euler himself already noted the covariance of the Euler-Lagrange equation under coordinate transformations (see [6], Chap. 4), Weierstrass introduced fields [7, 8] and Carathéodory formulated his "royal road" approach using equivalent variational problems [9]. It appears however that the natural idea to view the field of extremals as a coordinate transformation is a new contribution to the classical theory.

\section{Equivalences of Variational Problems}

We discuss Leitmann's method of transforming a calculus of variations problem into an equivalent form [1, 2], which was complemented by the discussion in [10]. We shall discuss this method in the context of minimizing a functional

$$
J(x)=\int_{a}^{b} L(t, x, \dot{x}) \mathrm{d} t,
$$

where $x(t)$ is a vector depending continuously and piecewise continuous differentiable on $t \in[a, b] \subset \mathbb{R}, x \in \operatorname{PC}^{1}\left([a, b], \mathbb{R}^{m}\right)$. That is, there are points $a=t_{1}<\cdots<$ $t_{n}=b$ such that, on the intervals $\left(t_{i}, t_{i+1}\right), i=1, \ldots, n-1$, the derivative $\dot{x}$ exists and is a differentiable function. The function $L$ is assumed to be at least $C^{3}$ on the open set $O \times \mathbb{R}^{m} \subset \mathbb{R} \times \mathbb{R}^{m} \times \mathbb{R}^{m}$. The case of fixed boundary conditions $x(a)=\alpha$ and $x(b)=\beta$ is investigated first; subsequently, the extension to free boundary conditions is made.

The derivative of a function $L$ with respect to a variable $x$ is indicated by $L_{x}$; that is, $L_{x} \xi=\sum_{i=1}^{m} L_{x_{i}} \xi^{i}$; a consequence of this notation is that, if $L=L(t, x, v)$, then

$$
L_{x v} \xi \eta=\sum_{i=1}^{m} \sum_{j=1}^{m} L_{x_{i} v_{j}} \xi^{j} \eta^{i}=\sum_{j=1}^{m} \sum_{i=1}^{m} L_{v_{j} x_{i}} \eta^{i} \xi^{j}=L_{v x} \eta \xi .
$$

Attention is restricted to the case that, for fixed values of $(t, x)$, the map $v \mapsto$ $L(t, x, v)$ has a positive-definite Hessian matrix $L_{v v}(t, x, v)$.

\subsection{Two Notions of Equivalence}

Leitmann's equivalence notion generalizes the notion of equivalent variational problems introduced by Carathéodory [9].

Let two points $(a, \alpha),(b, \beta) \in \mathbb{R} \times \mathbb{R}^{m}$ be given, as well as an open and simply connected set $R \subset(a, b) \times \mathbb{R}^{m}$ such that $(a, \alpha)$ and $(b, \beta)$ are contained in the closure $\bar{R}$ of $R$ and such that $\bar{R} \subset O$. We introduce the sets $R_{t}=\left\{x \in \mathbb{R}^{m} \mid(t, x) \in R\right\}$ and note that $R_{t}$ is open for every $t \in(a, b)$. Define

$$
\mathcal{A}=\left\{x \in \mathrm{PC}^{1}\left([a, b], \mathbb{R}^{m}\right) \mid x(t) \in R_{t} \text { for all } t \in(a, b), x(a)=\alpha, x(b)=\beta\right\} .
$$

Moreover, let another open set $R^{*} \subset(a, b) \times \mathbb{R}^{m}$ and a diffeomorphism $\Xi$ be given which maps $R^{*}$ and an open set $O^{*}$ containing $R^{*}$ diffeomorphically onto $R$ and $O$ 
respectively and which is such that

$$
\Xi(t, x)=(t, \xi(t, x)) .
$$

The conditions on $\Xi$ will be weakened somewhat in Sect. 4. Finally, let $\alpha^{*}$ and $\beta^{*}$ be such that $\xi\left(a, \alpha^{*}\right)=\alpha, \xi\left(b, \beta^{*}\right)=\beta$, and define

$$
\mathcal{A}^{*}=\left\{y \in \mathrm{PC}^{1}\left([a, b], \mathbb{R}^{m}\right) \mid y(t) \in R_{t}^{*} \text { for all } t, y(a)=\alpha^{*}, y(b)=\beta^{*}\right\} .
$$

Define the operator $\mathcal{X}: \mathcal{A}^{*} \rightarrow \mathcal{A}$ by setting $(\mathcal{X} y)(t)=\xi(t, y(t))$ and note that $\mathcal{X}$ maps $\mathcal{A}^{*}$ bijectively onto $\mathcal{A}$.

Definition 2.1 Let $\Xi, \mathcal{A}, \mathcal{A}^{*}$ be as above. The functionals $J: \mathcal{A} \rightarrow \mathbb{R}$ and $J^{*}: \mathcal{A}^{*} \rightarrow \mathbb{R}$, where

$$
J(x)=\int_{a}^{b} L(t, x, \dot{x}) \mathrm{d} t \quad \text { and } \quad J^{*}(y)=\int_{a}^{b} L^{*}(t, y, \dot{y}) \mathrm{d} t,
$$

are Leitmann equivalent (by $\Xi$ ) if there is a $C^{1}$ function $S^{*}: O^{*} \rightarrow \mathbb{R}$ such that the equation

$$
L\left(t, \xi, \xi_{t}+\xi_{y} \dot{y}\right)=L^{*}(t, y, \dot{y})+S_{t}^{*}(t, y)+S_{y}^{*}(t, y) \dot{y}
$$

holds identically in $(t, y, \dot{y}) \in O^{*} \times \mathbb{R}^{m}$. The two functionals are Carathéodory equivalent if (5) holds with $\Xi$ being the identity map.

We have the following well-known theorem $[1,9,10]$; in the context of this paper, the proof bears repeating.

Theorem 2.1 If $J$ and $J^{*}$ are Leitmann equivalent by $\Xi$, then a minimizer $\bar{y}$ of $J^{*}$ gives rise to a minimizer $\bar{x}$ of $J$ by setting $\bar{x}(t)=\xi(t, \bar{y}(t))$.

Proof Let the function $\bar{y}$ minimize $J^{*}$ over $\mathcal{A}^{*}$. Note that $\bar{x}=\mathcal{X} \bar{y}$; we want to show that $\bar{x}$ minimizes $J$ over $\mathcal{A}$. For this, pick any $x \in \mathcal{A}$ and let $y=\mathcal{X}^{-1} x$. Compute

$$
\begin{aligned}
J(x) & =\int_{a}^{b} L(t, x, \dot{x}) \mathrm{d} t=\int_{a}^{b}\left(L^{*}(t, y, \dot{y})+\frac{\mathrm{d}}{\mathrm{d} t} S^{*}(t, y)\right) \mathrm{d} t \\
& =J^{*}(y)+S^{*}\left(b, \beta^{*}\right)-S^{*}\left(a, \alpha^{*}\right) \geq J^{*}(\bar{y})+S^{*}\left(b, \beta^{*}\right)-S^{*}\left(a, \alpha^{*}\right) \\
& =\int_{a}^{b}\left(L^{*}(t, \bar{y}, \dot{\bar{y}})+\frac{\mathrm{d}}{\mathrm{d} t} S^{*}(t, \bar{y})\right) \mathrm{d} t=J(\bar{x}) .
\end{aligned}
$$

As $x$ was chosen arbitrarily, it follows that $J(x) \geq J(\bar{x})$ for all $x \in \mathcal{A}$.

\subsection{Equivalence of the Equivalences}

Given $J(x)=\int_{a}^{b} L(t, x, \dot{x}) \mathrm{d} t$ and a continuously differentiable function $\xi(t, x)$ such that $\xi(t,$.$) is a diffeomorphism, introduce$

$$
\hat{L}(t, y, \dot{y})=L\left(t, \xi(t, y), \xi_{t}(t, y)+\xi_{y}(t, y) \dot{y}\right)
$$


and consider the corresponding variational problem of minimizing

$$
\hat{J}(y)=\int_{a}^{b} \hat{L}(t, y, \dot{y}) .
$$

Let moreover

$$
J^{*}(y)=\int_{a}^{b} L^{*}(t, y, \dot{y}) \mathrm{d} t .
$$

As

$$
L(t, x, \dot{x})=\hat{L}(t, y, \dot{y}),
$$

it follows immediately that Leitmann equivalence by $\Xi$ of $J$ and $J^{*}$ is the same thing as Carathéodory equivalence of $\hat{J}$ and $J^{*}$.

\section{Simple Variational Problems}

The point of Leitmann's method is that, by taking appropriate coordinates, the transformed problem is particularly easy to solve [3]. This extends Carathéodory's "royal road" approach to field theory, which is now sketched briefly.

\subsection{Royal Road of Carathéodory}

In the royal road approach, the equivalent problem is required to satisfy the following. For every pair $(t, x)$, there is a vector $v(t, x)$ such that $\dot{x}=v(t, x)$ minimizes

$$
\dot{x} \mapsto L^{*}(t, x, \dot{x}) .
$$

Moreover, the minimum should be equal to 0 . If this is the case, it follows immediately that the integral curves of the differential equation

$$
\dot{x}=v(t, x)
$$

satisfy $J^{*}(x)=0$ and that this is indeed the smallest value possible; that is, the integral curves of (9) are absolute minimizers of $J$.

From the identity

$$
L^{*}(t, x, \dot{x})=L(t, x, \dot{x})-S_{t}(t, x)-S_{x}(t, x) \dot{x},
$$

the above conditions imply the Carathéodory fundamental equations

$$
\begin{aligned}
& L^{*}(t, x, \dot{x})=L(t, x, \dot{x})-S_{t}(t, x)-S_{x}(t, x) \dot{x}=0, \\
& L_{v}^{*}(t, x, \dot{x})=L_{v}(t, x, \dot{x})-S_{x}(t, x)=0,
\end{aligned}
$$

if $\dot{x}=v(t, x)$ minimizes $v \mapsto L^{*}(t, x, v)$. Introducing the costate $p=L_{v}(t, x, \dot{x})$ and the Hamilton function

$$
H(t, x, p)=\max _{\dot{x}}\{p \dot{x}-L(t, x, \dot{x})\}
$$


it follows that the fundamental equations are equivalent to the well-known relations

$$
p=S_{x}(t, x), \quad S_{t}+H\left(t, x, S_{x}\right)=0 ;
$$

the second of these is the Hamilton-Jacobi equation. The point of the Carathéodory approach is that, if the Hamilton-Jacobi equation can be solved, then the transformation to the equivalent system is possible and absolute minimizers are obtained.

\subsection{Royal Road of Leitmann}

Leitmann's approach simplifies the original variational problem by choosing the transformation $\xi$ as the inverse of a rectifying transformation of a field of extremals of the initial minimization problem. In the Leitmann approach, the Hamilton-Jacobi equation is also solved, but only implicitly.

By definition, the extremals are solutions of the Euler-Lagrange equation

$$
L_{x}-\frac{\mathrm{d}}{\mathrm{d} t} L_{v}=0
$$

As a differential equation of order $2 m$, the general solution $x=x(t, c)$ depends on $2 m$ parameters, the integration constants $c=\left(c_{1}, \ldots, c_{2 m}\right)$.

Assume that there is a value $c=\bar{c}$ such that the extremal $\bar{x}(t)=x(t, \bar{c})$ satisfies the boundary conditions $x(a)=\alpha, x(b)=\beta$. Let $Y \subset \mathbb{R}^{m}$ be a simply connected open set. Any continuously differentiable map $c: Y \rightarrow \mathbb{R}^{2 m}$ defines an $m$-parameter subfamily $\xi$ of extremals

$$
\xi=\xi(t, y)=x(t, c(y)) .
$$

The subfamily $\xi$ embeds the extremal $\bar{x}$ if $c(\bar{y})=\bar{c}$ for some $\bar{y} \in Y$. To introduce the notion of a field of extremals, define first the covectors

$$
\eta=\eta(t, y)=L_{v}\left(t, \xi(t, y), \xi_{t}(t, y)\right)
$$

Technically speaking, the element $(\xi, \eta)$ is a point in the cotangent bundle $T^{*} \mathbb{R}^{m}$; for the purposes of this paper, $T^{*} \mathbb{R}^{m}=\mathbb{R}^{m} \times \mathbb{R}^{m}$; in what follows, we shall be using the bundle notation. Assume that the vectors $v_{i}=\left(\xi_{y_{i}}, \eta_{y_{i}}\right), i=1, \ldots, m$, are linearly independent for all $y$; then the set

$$
\mathcal{F}_{t}=\left\{(x, p) \in T^{*} \mathbb{R}^{m} \mid x=\xi(t, y), p=\eta(t, y), y \in Y\right\}
$$

is an immersed $m$-dimensional submanifold of $T^{*} \mathbb{R}^{m}$ for every $t \in[a, b]$. Note that the tangent space to $\mathcal{F}_{t}$ at a point $\left(x_{0}, p_{0}\right)=\left(\xi\left(t, y_{0}\right), \eta\left(t, y_{0}\right)\right)$ is spanned by

$$
v_{j}=\left(\xi_{y_{j}}\left(t, y_{0}\right), \eta_{y_{j}}\left(t, y_{0}\right)\right), \quad j=1, \ldots, m \text {. }
$$

If $\omega=\mathrm{d} p \wedge \mathrm{d} x$ is the canonical 2-form [11], then

$$
\omega\left(v_{i}, v_{j}\right)=\eta_{y_{i}} \xi_{y_{j}}-\eta_{y_{j}} \xi_{y_{i}}
$$


Carathéodory uses the older "Lagrange bracket" notation $\left[y_{i}, y_{j}\right]$ instead of $\omega\left(v_{i}, v_{j}\right)$. Recall that a submanifold $\mathcal{M} \subset T^{*} \mathbb{R}^{m}$ is called Lagrangian if $\omega=0$ on $\mathcal{M}$; that is, $\mathcal{F}_{t}$ is Lagrangian if all the Lagrange brackets vanish identically on $\mathcal{F}_{t}$. Actually, the vanishing of the Lagrange brackets has only to be verified for a single value of $t$.

Theorem 3.1 If $\mathcal{F}_{t_{0}}$ is Lagrangian, then $\mathcal{F}_{t}$ is Lagrangian for all $t$.

For completeness sake, the proof of this theorem is given in the Appendix.

Definition 3.1 Let $\xi:[a, b] \times Y \rightarrow \mathbb{R}^{m}$ be an $m$-parameter subfamily of extremals. The family $\xi$ defines an extremal field of the minimization problem if the following conditions hold:

(i) For $t \in(a, b)$, the map $y \mapsto \xi(t, y)$ is a diffeomorphism from $Y$ onto its image; that is, $\operatorname{det} \xi_{y}(t, y) \neq 0$ for all $(t, y) \in(a, b) \times Y$ and $\xi\left(t, y_{1}\right) \neq \xi\left(t, y_{2}\right)$ for any $t \in(a, b)$. In particular, no extremals of the family intersect.

(ii) The manifold $\mathcal{F}_{t}$ is Lagrangian for some $t \in[a, b]$; equivalently, all the Lagrange brackets vanish identically in $t$.

In this paper, a field is called regular if $\xi(t,$.$) is a C^{2}$ diffeomorphism onto its image.

In the following, typically $R^{*} \subset(a, b) \times Y$ and $R \subset \Xi((a, b) \times Y)$; that is, the fields of extremals $t \mapsto \xi(t, y)$ with $y \in Y$ cover $R$. Moreover, note that the region $R^{*}$ is simply connected, as it is the diffeomorphic image of the simply connected set $R$.

If $\xi(t, y)$ is a regular field, then the inverse of $(t, x)=\Xi(t, y)$ is a rectifying transformation of the field; see Fig. 1, where any extremal $x(t)=\xi(t, y)$ corresponds to a constant value of $y$. Figure 1 suggests strongly that, if to transform the problem a field $\xi$ is used that embeds an extremal satisfying the boundary conditions, then the equivalent variational problem has simple solutions.

To make this precise, let

$$
\hat{L}(t, y, \dot{y})=L\left(t, \xi(t, y), \xi_{t}(t, y)+\xi_{y}(t, y) \dot{y}\right)
$$

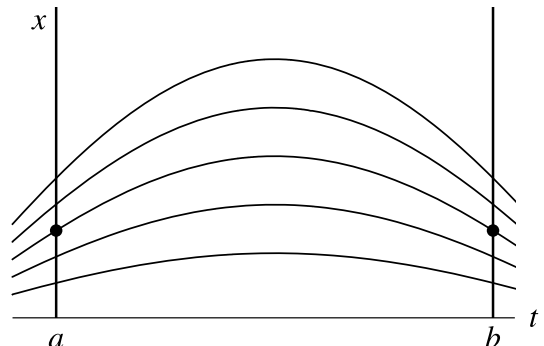

(a) Field of extremals

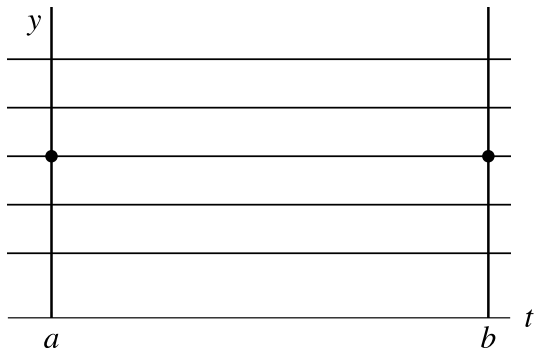

(b) Rectified field of extremals

Fig. 1 Taking for the Leitmann transformation the inverse of a rectifying transformation of a field of extremals that embeds an extremal satisfying the boundary conditions, the equivalent Leitmann problem simplifies 
and expand this expression in the last argument around $\xi_{t}(t, y)$. The Taylor theorem yields that

$$
\hat{L}(t, y, \dot{y})=L\left(t, \xi, \xi_{t}\right)+L_{v}\left(t, \xi, \xi_{t}\right) \xi_{y} \dot{y}+\ell(t, y, \dot{y}) \dot{y}^{2}
$$

where

$$
\ell(t, y, \dot{y})=(1 / 2) L_{v v}\left(t, \xi, \xi_{t}+\vartheta \xi_{y} \dot{y}\right) \xi_{y}^{2}
$$

for $0<\vartheta=\vartheta(t, y)<1$.

Introduce the functions

$$
s^{0}(t, y)=L\left(t, \xi, \xi_{t}\right)
$$

and

$$
s^{i}(t, y)=L_{v}\left(t, \xi, \xi_{t}\right) \xi_{y_{i}}=\eta \xi_{y_{i}}, \quad i=1, \ldots, m .
$$

The following observation goes back to Beltrami and Hilbert ([5], p. 396).

Theorem 3.2 There is a $C^{2}$ function $S^{*}(t, y)$ such that

$$
S_{t}^{*}=s^{0}, \quad S_{y_{i}}^{*}=s^{i}, \quad i=1, \ldots, m .
$$

Proof This is an integrability statement; since the domain of definition $R^{*}$ of $\xi$ is simply connected, the function $S^{*}$ exists if the implied mixed partials of $S^{*}$ are equal. We have

$$
s_{y_{i}}^{0}=L_{x} \xi_{y_{i}}+L_{v} \xi_{t y_{i}}, \quad s_{t}^{i}=\left(\frac{\mathrm{d}}{\mathrm{d} t} L_{v}\right) \xi_{y_{i}}+L_{v} \xi_{y_{i}}, \quad s_{y_{j}}^{i}=\eta_{y_{i}} \xi_{y_{j}}+\eta \xi_{y_{i} y_{j}} .
$$

The equation

$$
s_{y_{i}}^{0}-s_{t}^{i}=\left(L_{x}\left(t, \xi, \xi_{t}\right)-\frac{\mathrm{d}}{\mathrm{d} t} L_{v}\left(t, \xi, \xi_{t}\right)\right) \xi_{y_{i}}=0
$$

holds by virtue of $t \mapsto \xi(t, y)$ satisfying the Euler-Lagrange equation. Moreover

$$
s_{y_{j}}^{i}-s_{y_{i}}^{j}=\eta_{y_{i}} \xi_{y_{j}}-\eta_{y_{j}} \xi_{y_{i}}+\eta\left(\xi_{y_{i} y_{j}}-\xi_{y_{j} y_{i}}\right)=0
$$

by the vanishing of the Lagrange brackets and the equality of the mixed partial derivatives.

Combining this result with (19) yields that there is a $C^{2}$ function $S^{*}=S^{*}(t, y)$ such that

$$
L\left(t, \xi, \xi_{t}+\xi_{y} \dot{y}\right)=S_{t}^{*}+S_{y}^{*} \dot{y}+\ell(t, y, \dot{y}) \dot{y}^{2} .
$$

We remark that solving the Hamilton-Jacobi equation in the Carathéodory approach reduces to integrating $\dot{S}=L$ along extremals. As in the Leitmann approach the extremals are rectified, this equation is equivalent to $S_{t}^{*}(t, y)=\hat{L}(t, y, 0)$.

It is a corollary to Theorem 3.2 that, if $\Xi$ is taken to be the inverse of a rectifying transformation of a regular field of extremals, then the original variational problem is Leitmann equivalent, by $\Xi$, to a problem that can be solved by inspection. 
Theorem 3.3 Assume that there is a regular field $\xi$ of extremals of $J=\int_{a}^{b} L \mathrm{~d} t$ that covers $R$ and that the associated map $\Xi(t, y)=(t, \xi(t, y))$ is a diffeomorphism on an open set $O^{*}$ containing $R^{*}$. Then, $J$ is Leitmann equivalent by $\Xi$ to

$$
J^{*}(y)=\int_{a}^{b} \ell(t, y, \dot{y}) \dot{y}^{2} \mathrm{~d} t .
$$

Proof This is a direct corollary of Theorem 3.2.

Recall the assumption that $L$ is regular, that is, $v \mapsto L_{v v}(t, x, v)$ is positive definite for all $(t, x) \in R$.

Theorem 3.4 Under the same assumptions as in Theorem 3.3, if $\xi$ embeds an extremal $\bar{x}$ in the form $\bar{x}(t)=\xi(t, \bar{y})$, with constant $\bar{y}$, then $\bar{x}$ is the unique minimizer of $J$ in $\mathcal{A}$.

Proof This can be achieved by inspection from Theorem 3.3.

To summarize, if $\xi$ can be taken as the inverse of a regular rectifying transformation of a field that embeds an extremal satisfying the boundary conditions, the original variational problem is Leitmann equivalent to a problem whose minimum can be determined by inspection.

\subsection{Example}

As an example, consider the problem of minimizing

$$
J(x)=\int_{-1}^{1}\left(\dot{x}^{2}-x^{2}\right) \mathrm{d} t,
$$

subject to the boundary conditions $x(-1)=x(1)=1$.

The Euler-Lagrange equation for this problem reads as

$$
\ddot{x}+x=0 .
$$

The general solution to this differential equation is $x(t)=c_{1} \cos t+c_{2} \sin t$; the single solution $\bar{x}$ that satisfies the boundary conditions is obtained by setting $c_{1}=1 / \cos 1$ and $c_{2}=0$.

A field of extremals embedding $\bar{x}$ is given by $\xi(t, y)=y \cos t$. Transforming the functional by $\Xi(t, y)=(t, y \cos t)$ leads to

$$
\hat{J}(t, y, \dot{y})=\int_{-1}^{1}\left(\dot{y}^{2} \cos ^{2} t-y^{2} \cos 2 t-y \dot{y} \sin 2 t\right) \mathrm{d} t,
$$

with transformed boundary conditions $y(-1)=y(1)=1 / \cos 1$. The function $S$ reads as

$$
S(t, y)=-\frac{y^{2}}{2} \sin 2 t
$$


Using $S$ in the equivalence definitions and posing

$$
J^{*}(y)=\int_{-1}^{1} \ell(t, y, \dot{y}) \dot{y}^{2} \mathrm{~d} t=\int_{-1}^{1} \dot{y}^{2} \cos ^{2} t \mathrm{~d} t,
$$

the functional $J$ is seen to be Leitmann equivalent, and $\hat{J}$ to be Carathéodory equivalent, to the functional $J^{*}$.

Note that $\ell>0$ for $t \in(-1,1)$. By inspection, we see that the function $\bar{y}(t)=$ $1 / \cos 1$ is the unique minimizer of $J^{*}$; consequently,

$$
\bar{x}(t)=\frac{\cos t}{\cos 1}
$$

is the unique minimizer of $J$.

\section{Boundary Conditions}

In this section, different types of boundary conditions are considered.

\subsection{Fixed Endpoints}

Consider again the fixed endpoint problem to minimize $J$, where

$$
J(x)=\int_{a}^{b} L(t, x, \dot{x}) \mathrm{d} t, \quad x(a)=\alpha, \quad x(b)=\beta .
$$

Recall the idea of a central field: this is a family of extremals that all pass through a single point $(a, \alpha)$. Such a family obviously embeds any extremal satisfying the boundary conditions. Moreover, it has automatically the field property.

Theorem 4.1 Let $Y \subset \mathbb{R}^{m}$ be an open simply connected set, let $R^{*}=(a, b) \times Y$, and let $\xi: R^{*} \rightarrow \mathbb{R}^{m}$ be an m-parameter family of extremals such that, for $t \in(a, b)$, the map $y \mapsto \xi(t, y)$ maps $Y$ diffeomorphically onto its image and with the property that, for all $y \in Y$,

$$
\lim _{t \downarrow a} \xi(t, y)=\alpha .
$$

Then, $\xi$ is a field of extremals; it is called the central field around $(a, \alpha)$.

Note that $\Xi: R^{*} \rightarrow R$ cannot be extended diffeomorphically to an open set $O^{*}$ containing $R^{*}$, since $y \mapsto \xi(t, y)$ fails to be one-to-one if $t=a$. Therefore, the definitions of the sets $\mathcal{A}$ and $\mathcal{A}^{*}$ and the definition of Leitmann equivalence have to be adapted.

The new definitions of $\mathcal{A}$ and $\mathcal{A}^{*}$ are

$$
\mathcal{A}=\left\{x \in \mathrm{PC}^{1}\left((a, b), \mathbb{R}^{m}\right) \mid x(t) \in R_{t} \text { for all } t \in(a, b), \lim _{t \downarrow a} x(t)=\alpha, \lim _{t \uparrow b} x(t)=\beta\right\},
$$




$$
\begin{gathered}
\mathcal{A}^{*}=\left\{y \in \mathrm{PC}^{1}\left((a, b), \mathbb{R}^{m}\right) \mid y(t) \in R_{t}^{*} \text { for all } t \in(a, b),\right. \\
\left.\lim _{t \downarrow a} \xi(t, y(t))=\alpha, \lim _{t \uparrow b} \xi(t, y(t))=\beta\right\} .
\end{gathered}
$$

The sharper version of Leitmann and Carathéodory equivalence is given in the following definition.

Definition 4.1 Let $\Xi, \mathcal{A}, \mathcal{A}^{*}$ be as introduced in this section. Moreover, let $R_{a}=$ $\{(a, \alpha)\}$ and $R_{b}=\{(b, \beta)\}$. The functionals $J: \mathcal{A} \rightarrow \mathbb{R}$ and $J^{*}: \mathcal{A}^{*} \rightarrow \mathbb{R}$, where

$$
J(x)=\int_{a}^{b} L(t, x, \dot{x}) \mathrm{d} t \quad \text { and } \quad J^{*}(y)=\int_{a}^{b} L^{*}(t, y, \dot{y}) \mathrm{d} t,
$$

are Leitmann equivalent (by $\Xi$ ) if there is a continuous function

$$
S: R \cup R_{a} \cup R_{b} \rightarrow \mathbb{R}
$$

such that $S$ is $C^{1}$ on $R$ and such that, if $S^{*}(t, y)=S(t, \xi(t, y))$, the equation

$$
L\left(t, \xi, \xi_{t}+\xi_{y} \dot{y}\right)=L^{*}(t, y, \dot{y})+S_{t}^{*}(t, y)+S_{y}^{*}(t, y) \dot{y}
$$

holds identically in $(t, y, \dot{y}) \in R \times \mathbb{R}^{m}$.

The two functionals are Carathéodory equivalent if (25) holds with $\Xi$ being the identity map.

Theorem 2.1 still holds if in the proof the quantities $S^{*}\left(a, \alpha^{*}\right)$ and $S^{*}\left(b, \beta^{*}\right)$ are replaced by $\lim _{t \downarrow a} S^{*}(t, y(t))=S(a, \alpha)$, etc.

Given a central field $\xi$ around $(a, \alpha)$ and using again Theorem 3.2, the problem of minimizing $J(x)$ is seen to be Leitmann equivalent by $\xi$ to the problem of minimizing

$$
\hat{J}(y)=S(b, \beta)-S(a, \alpha)+\int_{a}^{b} \ell(t, y, \dot{y}) \dot{y}^{2} \mathrm{~d} t,
$$

subject to the single boundary condition

$$
\lim _{t \uparrow b} \xi(t, y(t))=\beta .
$$

In other words, the transformation has changed an optimization problem with two fixed boundary conditions into one with only a single fixed boundary condition. Assuming that $y \mapsto \xi(b, y)$ is invertible and that $\xi\left(b, \beta^{*}\right)=\beta$, the boundary condition can be written as

$$
y(b)=\beta^{*} .
$$

Theorem 4.2 Let $J=\int_{a}^{b} L(t, x, \dot{x}) \mathrm{d} t$ with $L$ regular on $R$ and let $\xi$ be a regular central field covering $R$. If $y \mapsto \xi(t, y)$ is a diffeomorphism for $t=b$ and if $\bar{y}$ is such that

$$
\xi(b, \bar{y})=\beta,
$$

then the function $\bar{x}(t)=\xi(t, \bar{y})$ is the unique minimizer of $J$. 
Proof Since $\xi$ is a central field, we have that, for all $y$,

$$
\lim _{t \downarrow a} \xi(t, y)=\alpha \quad \text { and } \quad \lim _{t \downarrow a} \xi_{y}(t, y)=0 .
$$

By (21), it follows that $\lim _{t \downarrow a} S_{y}^{*}(t, y)=0$ and that $S^{*}(a, y)=C$ does not depend on $y$. It follows that the function $S: R \cup\{(a, \alpha),(b, \beta)\} \rightarrow \mathbb{R}$ is a well-defined continuous function if we set $S(t, \xi(t, y))=S^{*}(t, y)$ on $R^{*}$; in particular $S(a, \alpha)=C$. Invoking Theorem 3.2, it is seen that $J$ is Leitmann equivalent to minimizing

$$
J^{*}(y)=S(b, \beta)-C+\int_{a}^{b} \ell(t, y, \dot{y}) \dot{y}^{2} \mathrm{~d} t .
$$

As $S(b, \beta)-C$ does not depend on $y$, it is seen by inspection that $J^{*}$ is minimized if $\dot{y}=0$, that is, $y(t)=\bar{y}$ for all $t$.

\subsection{Single Free Endpoint}

Consider next the free endpoint problem of minimizing

$$
J(x)=\int_{a}^{b} L(t, x, \dot{x}) \mathrm{d} t, \quad x(a)=\alpha, \quad x(b) \text { free. }
$$

It is obvious how to change the definitions of the sets $\mathcal{A}$ and $\mathcal{A}^{*}$. In the definition of Leitmann equivalence, the set $R_{b}$ has now to be taken as $\bar{R} \cap\left(\{b\} \times \mathbb{R}^{m}\right)$. A simple modification of the proof of Theorem 4.2 yields the following theorem.

Theorem 4.3 Let $J=\int_{a}^{b} L(t, x, \dot{x}) \mathrm{d} t$ with $L$ regular on $R$, and let $\xi$ be a regular central field covering $R$. Let $S^{*}=S^{*}(t, y)$ be the function whose existence has been proved in Theorem 3.2. If $y \mapsto \xi(b, y)$ is a diffeomorphism and if $\bar{y}$ minimizes $y \mapsto$ $S^{*}(b, y)$, then $\bar{x}(t)=\xi(t, \bar{y})$ minimizes $J$.

Proof The only difference from the proof of Theorem 4.2 is that (28) now reads as

$$
J^{*}(y)=S^{*}(b, y(b))-S(a, \alpha)+\int_{a}^{b} \ell(t, y, \dot{y}) \dot{y}^{2} \mathrm{~d} t .
$$

It is clear that this integral is minimized if $y(t)$ is always equal to a constant $\bar{y}$, implying $\dot{y}=0$ and that the value of that constant should minimize $y \mapsto S^{*}(b, y)$.

Note that, since $S_{y}=L_{v}$, the familiar necessary transversality condition $L_{v}=0$ follows as a corollary.

\subsection{Endpoint on a Manifold}

The extension to the case of a fixed initial point $(a, \alpha)$ and an endpoint located on some manifold is straightforward. 
Let

$$
J(x)=\int_{a}^{b} L(t, x, \dot{x}) \mathrm{d} t
$$

and let $\xi$ be a central field of $L$ through the point $(a, \alpha)$. Moreover, let $\Gamma$ be an embedded submanifold of $\mathbb{R} \times \mathbb{R}^{m}$ contained in the boundary of $R$ and assume that $\xi$ covers an open set $U$ such that $R \cup \Gamma \subset U$. Let $\Gamma^{*}=\Xi^{-1}(\Gamma)$ be the diffeomorphic image of $\Gamma$ under the inverse of $\Xi$.

The problem is to minimize $J$ over

$$
\mathcal{A}=\left\{x \in \mathrm{PC}^{1}\left((a, b), \mathbb{R}^{m}\right) \mid x(t) \in R_{t} \text { for all } t \in(a, b), \lim _{t \downarrow a} x(t)=\alpha,(b, x(b)) \in \Gamma\right\} .
$$

Set also

$$
\begin{gathered}
\mathcal{A}^{*}=\left\{y \in \mathrm{PC}^{1}\left((a, b), \mathbb{R}^{m}\right) \mid y(t) \in R_{t}^{*} \text { for all } t \in(a, b), \lim _{t \downarrow a} \xi(t, y(t))=\alpha,\right. \\
\Xi(b, y(b)) \in \Gamma\} .
\end{gathered}
$$

Theorem 4.4 Under the given hypotheses, let $S^{*}=S^{*}(t, y)$ be the function whose existence has been proved in Theorem 3.2. If $(\bar{t}, \bar{y}) \in \Gamma^{*}$ minimizes $S^{*}$ over $\Gamma^{*}$, then $\bar{x}(t)=\xi(t, \bar{y})$ defined on $(a, \bar{t})$ minimizes $J$ over $\mathcal{A}$.

Proof In this case, (28) reads as

$$
J^{*}(y)=S^{*}(b, y(b))-S(a, \alpha)+\int_{a}^{b} \ell(t, y, \dot{y}) \dot{y}^{2} \mathrm{~d} t,
$$

where $(b, y(b)) \in \Gamma^{*}$. It is clear that this integral is minimized if $y(t)$ is always equal to a constant $\bar{y}$, implying $\dot{y}=0$, and that the value of that constant should minimize $S^{*}(t, y)$ restricted to $\Gamma^{*}$.

\subsection{Double Free Endpoint}

Finally, consider the problem of minimizing

$$
J(x)=\int_{a}^{b} L(t, x, \dot{x}) \mathrm{d} t,
$$

with free endpoint conditions on both sides. The modifications of the definitions of $\mathcal{A}$, $\mathcal{A}^{*}, R_{a}, R_{b}$ are left to the reader.

Theorem 4.5 Let $Y \subset \mathbb{R}^{m}$ be an open simply connected set and let $\xi:(a, b) \times$ $Y \rightarrow \mathbb{R}^{m}$ be an m-parameter family of extremals such that, for $t \in(a, b)$, the map $y \mapsto \xi(t, y)$ maps $Y$ diffeomorphically onto its image and with the property that, for all $y \in Y$,

$$
\lim _{t \downarrow a} \eta(t, y)=0 .
$$

Then, $\xi$ is a field; we shall call it the field that is transversal at $t=a$. 
Proof The proof is completely analogous to that of Theorem 4.1, except that, instead of $\xi_{y_{i}}=0$, here $\eta_{y_{i}}(a, y)=0$ for all $y$ and all $i$.

Theorem 4.6 Let $J=\int_{a}^{b} L(t, x, \dot{x}) \mathrm{d} t$, with $L$ regular on $R$, and let $\xi$ be a regular field, transversal at $t=a$, covering $R$. Let $S^{*}=S^{*}(t, y)$ be the function whose existence has been proved in Theorem 3.2. If $y \mapsto \xi(b, y)$ is a diffeomorphism and if $\bar{y}$ minimizes $y \mapsto S^{*}(b, y)$, then $\bar{x}(t)=\xi(t, \bar{y})$ minimizes $J$.

Proof The proof is parallel to the proof of Theorem 4.3, with some modifications. Since $\xi$ is a transversal field, we have that, for all $y$,

$$
\lim _{t \downarrow a} S_{y}(t, y)=L_{v}=0 .
$$

It follows that $S(a, y)=C$ does not depend on $y$. To minimize $J$ is therefore equivalent to

$$
J^{*}(y)=S(b, y(b))-C+\int_{a}^{b} \ell(t, y, \dot{y}) \dot{y}^{2} \mathrm{~d} t .
$$

This integral is clearly minimized by a constant function $y(t)=\bar{y}$ such that $\bar{y}$ minimizes $y \mapsto S(b, y)$.

Open Access This article is distributed under the terms of the Creative Commons Attribution Noncommercial License which permits any noncommercial use, distribution, and reproduction in any medium, provided the original author(s) and source are credited.

\section{Appendix: Proof of Theorem 3.1}

Let us compute

$$
\frac{\mathrm{d}}{\mathrm{d} t} \omega\left(v_{i}, v_{j}\right)=\frac{\mathrm{d}}{\mathrm{d} t}\left(\eta_{y_{i}} \xi_{y_{j}}-\eta_{y_{j}} \xi_{y_{i}}\right)=\eta_{y_{i} t} \xi_{y_{j}}+\eta_{y_{i}} \xi_{y_{j} t}-\eta_{y_{j}} \xi_{y_{i}}-\eta_{y_{j}} \xi_{y_{i} t} .
$$

To evaluate this expression, the quantities $\eta_{y_{i}}$ and $\eta_{t y_{i}}$ have to be determined. Deriving (15) with respect to $y_{i}$ yields

$$
\eta_{y_{i}}=L_{v x} \xi_{y_{i}}+L_{v v} \xi_{t y_{i}}
$$

Since $\xi=\xi(t, y)$ is a family of extremals, the equation

$$
L_{x}\left(t, \xi, \xi_{t}\right)-\frac{\mathrm{d}}{\mathrm{d} t} L_{v}\left(t, \xi, \xi_{t}\right)=L_{x}\left(t, \xi, \xi_{t}\right)-\frac{\mathrm{d}}{\mathrm{d} t} \eta(t, y)=0
$$

holds identically in $y$. Deriving with respect to $y_{i}$ and rearranging terms yields

$$
\eta_{t y_{i}}=L_{x x} \xi_{y_{i}}+L_{x v} \xi_{t y_{i}}
$$

Substitution of (34) and (36) into (33) yields

$$
\frac{\mathrm{d}}{\mathrm{d} t} \omega\left(v_{i}, v_{j}\right)=\left(L_{x x} \xi_{y_{i}}+L_{x v} \xi_{t y_{i}}\right) \xi_{y_{j}}+\left(L_{v x} \xi_{y_{i}}+L_{v v} \xi_{t y_{i}}\right) \xi_{y_{j} t}
$$




$$
\begin{gathered}
-\left(L_{x x} \xi_{y_{j}}+L_{x v} \xi_{t y_{j}}\right) \xi_{y_{i}}-\left(L_{v x} \xi_{y_{j}}+L_{v v} \xi_{t y_{j}}\right) \xi_{y_{i} t} \\
=L_{x v} \xi_{t y_{i}} \xi_{y_{j}}+L_{v x} \xi_{y_{i}} \xi_{y_{j} t}-L_{x v} \xi_{t y_{j}} \xi_{y_{i}}-L_{v x} \xi_{y_{j}} \xi_{y_{i} t}=0 .
\end{gathered}
$$

The last equality holds by virtue of (2).

\section{References}

1. Leitmann, G.: On a class of direct optimization problems. J. Optim. Theory Appl. 108, 467-481 (2001)

2. Leitmann, G.: A note on absolute extrema of certain integrals. Int. J. Non-linear Mech. 2, 55-59 (1967)

3. Carlson, D., Leitmann, G.: Fields of extremals and sufficient conditions for the simplest problem of the calculus of variations. J. Glob. Optim. 40, 41-50 (2008)

4. Carlson, D., Leitmann, G.: Fields of extremals and sufficient conditions for the simplest problem of the calculus of variations in $n$-variables. Preprint (2008)

5. Giaquinta, M., Hildebrandt, S.: Calculus of Variations I. Grundlehren der mathematischen Wissenschaften, vol. 310. Springer, Heidelberg (1996)

6. Euler, L.: Methodus inveniendi lineas curvas maximi minimive proprietate gaudentes, sive solutio problematis isoperimetrici latissimo sensu accepti. Bousquet, Lausannae et Genevae (1744)

7. Kneser, A.: Lehrbuch der Variationsrechnung. Vieweg, Braunschweig (1900)

8. Weierstrass, K.: Vorlesungen über Variationsrechnung. Werke, vol. 7. Akademische Verlagsgesellschaft, Leipzig (1927)

9. Carathéodory, C.: Variationsrechnung und partielle Differentialgleichungen erster Ordnung. Teubner, Berlin (1935)

10. Carlson, D.: An observation on two methods of obtaining solutions to variational problems. J. Optim. Theory Appl. 114, 345-361 (2002)

11. Arnol'd, V.: Mathematical Methods of Classical Mechanics. Springer, Heidelberg (1989) 